\title{
Stress, itch and quality of life in chronic urticaria females
}

\author{
Alicja Ograczyk-Piotrowska, Zofia Gerlicz-Kowalczuk, Anna Pietrzak, Anna M. Zalewska-Janowska
}

Psychodermatology Department, Medical University of Lodz, Lodz, Poland

Adv Dermatol Allergol 2018; XXXV (2): 156-160

DOI: https://doi.org/10.5114/ada.2018.75237

\begin{abstract}
Introduction: Chronic urticaria (CU) belongs to a group of psychodermatological disorders, thus stress can play a significant role in this dermatosis onset and/or exacerbation. On the other hand, the disease itself accompanied by itch, may be a source of distress and could worsen patients' quality of life (QoL).

Aim: The first goal of our study was to compare stress intensity between CU subjects and the control group. The second aim was to investigate the relationships between disease-related parameters (CU severity, itch) and psychological variables (stress and QoL) in CU patients.

Material and methods: Forty-six female patients with CU participated in our study. Thirty-three healthy females constituted a control group. The following methods were applied: Urticaria Activity Score (UAS), Itch Severity Evaluation Questionnaire, Visual analogue scale (VAS), Social Readjustment Rating Scale (SRRS) and the Chronic Urticaria Quality of Life Questionnaire (CU-Q2oL).

Results: Chronic urticaria patients demonstrated a significantly higher stress level in comparison to the control group $(z=2.699 ; p<0.001)$. Regarding the total pruritus score, all CU-Q2oL dimensions were affected, except for subscale swelling/mental status. The strongest link was revealed between global itch and QoL subscale embarrassment $(r=0.51, p<0.001)$. There were also statistically significant correlations between stress (VAS scale and SRRS) and QoL (all at least $p<0.05$ ).

Conclusions: Taking into account the significant pruritus contribution to QoL impairment, it would be worth employing itch-coping trainings in the CU group. As a consequence, feeling of self-control and self-efficacy could be enhanced, thus resulting in the well-being improvement.
\end{abstract}

Key words: chronic urticaria, itch, quality of life, embarrassment.

\section{Introduction}

Chronic urticaria (CU) accounts for about $1-4 \%$ of the general population. Chronic urticaria diagnosis is established when the disease lasts for at least 6 weeks. Recurrent, temporary hives, accompanied by itch, are characteristic skin lesions in urticaria [1, 2]. Itch is described as a subjective and unpleasant sensation which results in scratching $[3,4]$. Of importance, evaluation of CU intensity performed by Urticaria Activity Score (UAS) comprises also itch assessment [5].

Additionally, CU is also classified as a psychodermatological disorder. It was proved that factors, such as stress, can affect urticarial wheals onset and exacerbation $[6,7]$. Emotional distress can also be related to itch intensity [8]. Stress is often experienced as anxiety which activates the autonomic nervous system. As a result, the itch threshold is lowered [9], so its intensity may be moderated not only by disease severity, but also by psychological factors. It was observed that individual perception of itch plays an important role in disease management [10]. What is more, strong focus on this unpleasant sensation can intensify itch and subsequently triggers scratching which is treated as the most automatic reaction of coping. On one hand, scratching allows to reduce inner tension, giving a sense of relief. On the other hand, however, intensive scratching can lead to itch-and-scratch vicious cycle formation. Such situation is associated with loss of control and feeling of inefficacy in itch management [10]. Of note, this unpleasant sensation is generally regarded as more burdensome for patients than pain [11].

It seems to be quite clear why itch is perceived as one of the strongest symptoms with a negative influence on patients' quality of life (QoL) [10, 12]. Its intensity also plays an important role in worsening patients' well-being $[10,12]$. Moreover, itch exerts a negative impact on physical, cognitive, emotional and social functioning [7, 13-15].

Address for correspondence: Alicja Ograczyk-Piotrowska, Psychodermatology Department, Medical University of Lodz, 251 Pomorska St, 92-213 Lodz, Poland, phone: +48 502099 388, e-mail: alicja.ograczyk@umed.lodz.pl Received: 28.10.2016, accepted: 24.05.2017. 
It is worth emphasizing that evaluation of QoL in CU patients, by the most relevant tool, the Chronic Urticaria Quality of Life Questionnaire (CU-Q2oL), tackles limits related to restricted diet, pressure-free clothes, anti-allergenic cosmetics and methods of coping with treatment side effects, such as somnolence after anti-histamine drugs or mass weight gain due to steroids employment [16-18].

\section{Aim}

The first goal of our study was to compare stress intensity between CU subjects and the control group. The second aim was to investigate the relationships between disease-related parameters (CU severity and itch) and psychological variables (stress and QoL) in CU patients.

\section{Material and methods}

The final study group was composed of 46 females (age mean: $44.6 \pm 14.2$ years; range: $21-68$ years) after excluding male patients due to gender disproportions (only 5 of them took part in our research). All participants did not suffer from any dermatological conditions, apart from chronic inducible urticaria: dermographism 25 individuals, delayed pressure urticaria - 11 ones and cold urticarial - 10 participants. Almost one third of the group indicated a significant stress role in the course of their disease. The disease duration (median) was $11 \pm$ 10.4 months (from 1.5 months to 25 years). In order to compare stress level results, we recruited the control group composed of 33 healthy females (age mean: 46.3 \pm 14 .7; range: $18-78$ years) who consulted dermatologists because of common warts and/or melanocytic nevi. They did not suffer from any other dermatological or chronic/ infectious disorders.

All participants were dermatological and allergological patients (out- and in-patients) of the region.

The Bioethics Committee approval was obtained for the study and patients' informed consent forms were collected. The research was performed in agreement with the Helsinki Declaration.

The following methods were used in our study:

- authors' questionnaire comprising socio-demographic data.

- Urticaria Activity Score 7 (UAS 7) - the tool to assess urticaria severity. The scale is recommended by the EAACI/GA²LEN/EDF guidelines. Urticaria Activity Score was estimated as the total result of two scores (range: $0-6)$ : hives number (0-3) and itch intensity (0-3). The disease severity was examined over a period of 7 days to obtain weekly score (UAS 7) - range 1-42 points [5].

- Itch Severity Evaluation Questionnaire (by Szepietowski and Reich, 2010) - the scale to assess global itch and its characteristic features concerning range (one location, several locations, generalized itch (range:
0-3)), intensity (regarding scratching necessity with or without excoriations (range: 0-5)), episodes duration (short episodes (less than $10 \mathrm{~min}$ ), long episodes (longer than $10 \mathrm{~min}$ ) or constant itch (range: 0-5)) and sleep disturbances as the consequence of itch (range: 0-6). The global result (from 3 to 19) is calculated as the sum of all the subscales points [11].

- Visual analogue scale (VAS) - the scale was used to measure stress intensity (regarding last month). It is a $10 \mathrm{~cm}$ continuum (from " 0 " - no stress experienced to "10" - the most severe stress intensity). Results from 0 to 3 can be interpreted as mild, 3-6 as moderate, 7-9 as severe and 10 as very severe;

- Social Readjustment Rating Scale (SRRS) (by Holmes and Rahe, 1967) - the method to measure the stress level on the basis of the sum of stressful life events during last 12 months. The global result higher than 300 points can indicate the high life crisis level and the $80 \%$ risk of disease occurrence, 200-299- moderate life crisis level and the $50 \%$ probability of illness onset and 150-199 - mild life crisis level and the 35\% risk of disease development. The sum within the range of 0-150 points can be interpreted as lack of significant life problems [19].

- The Chronic Urticaria Quality of Life Questionnaire (CUQ2oL) (Polish adaptation by Brzoza et al., 2011) - the specific tool to measure QoL in CU patients. It consists of 23 items regarding the disease influence on individual functioning. The tool comprises six subscales: itching, swelling/mental status, functioning, sleep, eating/ limits and embarrassment. The results from subscales can be transferred for converted ones (range: 0-100) allowing for comparison between them. The higher the results, the more QoL is impaired [16, 20].

\section{Statistical analysis}

Statistica package (version 12) was employed for statistical analysis. Values such as: mean (M), median values (Me), minimum (min), maximum (max), standard deviation (SD), standard error of measurement (SEM) and percentiles (25-75\%) are demonstrated. The criterion of normal distribution was not met by some variables, so non-parametric tests - Mann-Whitney U-test ( $z$ ) and Spearman's rank correlation test $(r)$ were used. The statistical significance level was set at $p<0.05$.

\section{Results}

\section{Stress in studied groups}

The results concerning stress were compared between the study and the control group. CU patients experienced more intensive stress regarding subjective perception (VAS scale, $z$ test value $=2.699, p<0.01$ ). We did not observe any statistically significant difference be- 
Table 1. Quality of life in the study group (CU-Q2oL subscales)

\begin{tabular}{lccc}
\hline CU-Q2oL & Mean & Median & 25-75\% values \\
\hline Itching & 60.22 & 60.00 & $40.00-70.00$ \\
\hline Swelling/mental status & 49.39 & 48.00 & $36.00-60.00$ \\
\hline Functioning & 41.45 & 33.34 & $20.00-56.67$ \\
\hline Sleep & 51.63 & 52.50 & $30.00-70.00$ \\
\hline Eating/limits & 49.67 & 47.50 & $30.00-60.00$ \\
\hline Embarrassment & 62.61 & 70.00 & $40.00-80.00$ \\
\hline
\end{tabular}

tween the studied groups regarding the number of difficult events in participants' lives (SRRS scale, $p>0.05$ ).

\section{Disease severity, itch and quality of life}

The variables regarding disease severity, itch and quality of life could be analyzed only in the CU patients group. The median UAS 7 result was 35 (range: 7-42; SEM $=1.40$ ), which can be interpreted as the disease of severe intensity. Global itch results indicated its moderate level (mean: $8.00 \pm 4.15$; range: $3-19$ ). The majority of the group (48\%) suffered from itch concerning several locations and itch with the demand of scratching without excoriations ( $n=23 ; 50 \%$ of the patients). Short episodes of this unpleasant sensation were the most frequently reported ( $n=23 ; 50 \%$ of CU participants). Itch-related sleep disturbances were regarded by 18 patients (39\% of the group). Ten of them reported more than 3 awakenings during one night.

The quality of life results (expressed as converted scores) are presented in Table 1. Of note, subscales itching and embarrassment were the most severely affected.

Disease severity positively correlated with global itch score $(r=0.35, p<0.05)$, itch intensity $(r=0.33, p<0.05)$ and range $(r=0.33, p<0.05)$. Regarding psychological parameters, higher CU activity was associated with a higher stress level (VAS scale $-r=0.31, p<0.05$; SRRS $-r=0.33, p<0.05)$ and worse QoL (all dimensions). The strongest correlations were observed between disease severity and subscales itching $(r=0.61, p<0.001)$ and functioning $(r=0.48, p<0.05)$.

Any further relations, thus between age, disease duration and psychological variables were not revealed $(p>0.05)$.

\section{Stress, itch and quality of life - correlations}

We did not note any statistically significant correlations between stress (both scales) and itch (and its features). Regarding the global itch score, all CU-Q2oL dimensions were affected, except for subscale swelling/ mental status (results shown in Table 2).

We also observed statistically significant correlations between stress (VAS scale and SRRS) and QoL (results presented in Table 3).

\section{Discussion}

Bearing in mind the aim of our study, the differences between CU patients and the control group were noted. Chronic urticaria individuals demonstrated higher levels of stress, but this difference between research groups, was limited only to the subjective perception of this phenomenon (VAS scale). What is worth emphasizing, the statistical correlation was lost $(p>0.05)$ when we compared the amount of stressful events in participants' lives (the scale by Holmes and Rahe). So, the appraisal of the situations could be regarded as being of key relevance to the patients [21]. Additionally, some researchers reported that CU patients more often present specific personality traits, such as neuroticism [22, 23], which make them more sensitive to stress experience. As a consequence, patients could be more susceptible to CU relapses and exacerbations. This observation is consistent with literature data pointing to the role of emotional distress

Table 2. The correlations between itch and CU-Q2oL

\begin{tabular}{|c|c|c|c|c|c|c|c|c|c|c|c|c|}
\hline \multirow[t]{4}{*}{ CU-Q2oL } & \multicolumn{12}{|c|}{ Itch characteristics } \\
\hline & \multirow{2}{*}{\multicolumn{2}{|c|}{ Range }} & \multirow{2}{*}{\multicolumn{2}{|c|}{ Intensity }} & \multicolumn{6}{|c|}{ Frequency } & \multicolumn{2}{|c|}{ Global itch } \\
\hline & & & & & \multicolumn{2}{|c|}{$\begin{array}{l}\text { Short itch } \\
\text { episodes }\end{array}$} & \multicolumn{2}{|c|}{$\begin{array}{l}\text { Long itch } \\
\text { episodes }\end{array}$} & \multicolumn{2}{|c|}{ Constant itch } & & \\
\hline & $r$ & $p$ & $r$ & $p$ & $r$ & $p$ & $r$ & $p$ & $r$ & $p$ & $r$ & $p$ \\
\hline Itching & 0.17 & $>0.05$ & 0.22 & $>0.05$ & 0.25 & $>0.05$ & 0.24 & $>0.05$ & 0.25 & $>0.05$ & 0.44 & $<0.01$ \\
\hline $\begin{array}{l}\text { Swelling/mental } \\
\text { status }\end{array}$ & 0.22 & $>0.05$ & 0.24 & $>0.05$ & -0.04 & $>0.05$ & 0.07 & $>0.05$ & 0.17 & $>0.05$ & 0.28 & $>0.05$ \\
\hline Functioning & 0.26 & $>0.05$ & 0.19 & $>0.05$ & 0.33 & $<0.05$ & 0.24 & $>0.05$ & 0.25 & $>0.05$ & 0.45 & $<0.01$ \\
\hline Sleep & 0.09 & $>0.05$ & 0.33 & $<0.05$ & 0.20 & $>0.05$ & 0.31 & $<0.05$ & -0.06 & $>0.05$ & 0.35 & $<0.05$ \\
\hline Eating/limits & 0.15 & $>0.05$ & 0.29 & $>0.05$ & 0.13 & $>0.05$ & 0.18 & $>0.05$ & 0.19 & $>0.05$ & 0.42 & $<0.01$ \\
\hline Embarrassment & 0.23 & $>0.05$ & 0.44 & $<0.01$ & 0.17 & $>0.05$ & 0.18 & $>0.05$ & 0.31 & $<0.05$ & 0.51 & $<0.001$ \\
\hline
\end{tabular}

$r$-Spearman's rank correlation, $p$ - significance level. 
Table 3. The correlations between stress and CU-Q2oL

\begin{tabular}{lcccc}
\hline CU- $\mathrm{Q}_{2} \mathrm{OL}$ & \multicolumn{2}{c}{ Stress (VAS) } & \multicolumn{2}{c}{ Stress (SRRS) } \\
\cline { 2 - 5 } & $r$ & $p$ & $r$ & $p$ \\
\hline Itching & 0.38 & $<0.01$ & 0.28 & $>0.05$ \\
\hline Swelling/mental status & 0.42 & $<0.01$ & 0.46 & $<0.01$ \\
\hline Functioning & 0.23 & $>0.05$ & 0.26 & $>0.05$ \\
\hline Sleep & 0.35 & $<0.05$ & 0.35 & $<0.05$ \\
\hline Eating/limits & 0.05 & $>0.05$ & 0.36 & $<0.05$ \\
\hline Embarrassment & 0.25 & $>0.05$ & 0.33 & $>0.05$ \\
\hline r-Spearman's rank correlation, $p$ - significance level. & & & &
\end{tabular}

in the disease onset $[6,7,24-26]$. On the other hand, one's experience of difficult emotions can be also perceived as a disease consequence. The latter was revealed by CU-Q2oL. In other words, the examined group of CU patients manifested worsened QoL. This observation is in agreement with literature data [16, 18, 27-30]. The above seems to be explained by an unpredictable course of CU, recurrent disease episodes and the location of skin lesions (on visible body parts). Exposed hives could cause embarrassment in social situations and lead to stigmatization. This phenomenon is well documented regarding other dermatoses, for example in psoriatics or in individuals suffering from atopic dermatitis [31, 32]. In our research, the QoL subscale embarrassment was most affected.

Itch is the symptom strongly related to patients' wellbeing. Such associations were also noted in other studies $[10,12,15]$. Itch (its global result) was associated with all domains of QoL, apart from subscale swelling/mental status. The strongest correlation was marked between its total score and embarrassment. It could be explained on the basis of patients' reports indicating that itch leads directly to scratching which attracts negative attention of others and influence one's social perception. As the consequence, patients could feel ashamed in public situations [17]. It was even noted that people suffering from contact dermatitis avoided social interactions due to their burdensome itch and the demand of scratching related to it $[33,34]$. We could assume that such situations could lead to social interactions deficits in CU patients. Additionally, patients could pay their attentional focus to bodily sensations, what also enhances itch and scratching behaviour [35]. So, coping with it may become more difficult and could lead to belief of losing control over that symptom. Finally, feelings of helplessness can develop and be a source of worse QoL [10].

As for current study limitations, the number of the patients seems to be not large, but at this stage we were unable to extend the study. Furthermore, we did not take into account the significance of cognitive appraisal in perception of stressful situations and disease cop- ing strategies. Both parameters could play a significant mediating role, providing more detailed explanation of stress, itch and QoL relations.

\section{Conclusions}

Taking into consideration the significant role of itch in QoL impairment in CU patients, it would be worth employing itch-coping trainings in this group. The effectiveness of such interventions is also reported in literature data [36]. Better management of this sensation could result in breaking the itch-and-scratch vicious cycle. As a consequence, feeling of self-control and self-efficacy could develop, thus leading to QoL improvement in CU patients.

\section{Acknowledgments}

This study was supported by statutory grant of the Medical University of Lodz No. 503/1-137-04/503-01 and grant for Young Researches of the Medical University of Lodz No. 502-03/1-137-04/502-14-205.

\section{Conflict of interest}

The authors declare no conflict of interest.

\section{References}

1. Seneczko F, Kaszuba A. Allergic skin diseases [Polish]. In: Dermatology for Cosmetologists. Adamski Z, Kaszuba A (eds). Elsevier Urban \& Partner, Wrocław 2010; 65-77.

2. Jabłońska S, Chorzelski T. Skin diseases for medical students and doctors [Polish]. Wydawnictwo Lekarskie PZWL, Warsaw 1994.

3. Verhoeven EW, de Klerk S, Kraaitmaat FW, et al. Biopsychological mechanism of chronic itch in patients with skin diseases: a review. Acta Derm Venereol 2008; 88: 211-8.

4. Verhoeven EW, Kraaitmaat FW, van de Kerkhof PC, et al. Prevalence of physical symptoms of itch, pain and fatigue in patients with skin diseases in general practice. J Br Dermatol 2007; 156: 1346-9. 
5. Młynek A, Zalewska-Janowska A, Martus P, et al. How to assess disease activity in patients with chronic urticaria? Allergy 2008; 63: 777-80.

6. Conrad R, Geiser F, Haidl G, et al. Relationship between anger and pruritus perception in patients with chronic idiopathic urticaria and psoriasis. J Eur Acad Dermatol Venereol 2008; 22: 1062-9.

7. Zalewska-Janowska A. Psychodermatology in allergology [Polish]. Alerg Astma Immunol 2010; 15: 109-17.

8. Niemeier V, Nippesen M, Kupfer J, et al. Psychological factors associated with hand dermatoses: which subgroup needs additional psychological care? Br J Dermatol 2002; 146: 1031-7.

9. Seiffert K, Hilbert E, Schaechinger $\mathrm{H}$, et al. Psychophysiological reactivity under mental stress in atopic dermatitis. Dermatology 2005; 210: 286-93.

10. Tey HL, Wallengren J, Yosipovitch G. Psychosomatic factors in pruritus. Clin Dermatol 2013; 31: 31-40.

11. Szepietowski J, Reich A. Itch. Patomechanism, Clinics, Treatment [Polish]. Termedia Wydawnictwa Medyczne, Poznan 2010.

12. Verhoeven L, Kraaimaat FW, Duller P, et al. Cognitive, behavioral and physiological reactivity to chronic itching: analogies to chronic pain. Int J Behav Med 2006; 13: 237-43.

13. Yosipovitch G, Ansari N, Goon A, et al. Clinical characteristics of pruritus in chronic idiopathic urticaria. Br J Dermatol 2002; 147: 32-6.

14. Mathias SD, Dreskin SC, Kaplan A, et al. Development of a daily diary for patients with chronic idiopathic urticaria. Ann Allergy Asthma Immunol 2010; 105: 142-8.

15. O'Donnell BF. Urticaria: impact on quality of life and economic cost. Immunol Allergy Clin North Am 2014; 34: 89-104.

16. Brzoza Z, Badura-Brzoza K, Młynek A, et al. Adaptation and initial results of the Polish version of the GA2LEN Chronic Urticaria Quality Of Life Questionnaire (CU-O2oL). J Dermatol Sci 2011; 62: 36-41.

17. Młynek A, Magerl M, Hanna M, et al. The German version of the Chronic Urticaria Quality-of-Life Questionnaire: factor analysis, validation, and initial clinical findings. Allergy 2009; 64: 927-36.

18. Kocaturk E, Weller K, Martus P, et al. Turkish version of the Chronic Urticaria Quality of Life Questionnaire: cultural adaptation, assessment of reliability and validity. Acta Derm Venereol 2012; 92: 419-25.

19. Holmes TH, Rahe RH. The Social Readjustment Rating Scale. J Psychosom Res 1967; 11: 213-8.

20. Baiardini I, Pasquali M, Braido F, et al. A new tool to evaluate impact of chronic urticaria on quality of life: chronic urticaria quality of life questionnaire (CU-Q2oL). Allergy 2005; 60: 1073-8.

21. Heszen I, Sęk H. Health Psychology [Polish]. Wydawnictwo Naukowe PWN, Warsaw 2008.

22. Lyketsos GC, Stratogos GC, Tawil G, et al. Hostile personality characteristics, dysthymic states and neurotic symptoms in urticaria, psoriasis and alopecia. Psychother Psychosom 1985; 44: 122-31.

23. Ma LH, Tang LY, Luo GY, et al. A survey of psychosocial factors of psychosomatic diseases. Chin J Clin Psychol 2002; 10: 266-9.

24. Berrino AM, Voltolini S, Fiaschi D, et al. Chronic urticaria: importance of a medical-psychological approach. Eur Ann Allergy Clin Immun 2006; 38: 149-52.
25. Ozkan M, Oflaz SB, Kocaman N, et al. Psychiatric morbidity and quality of life in patients with chronic idiopathic urticaria. Ann Allergy Asthma Immunol 2007; 99: 29-33.

26. Yang HY, Sun CC, Wu YC, et al. Stress, insomnia and chronic idiopathic urticaria - a case control study. J Formos Med Assoc 2005; 104: 254-63.

27. Ucmak D, Akkurt M, Ucmak F, et al. Investigation of dermatology life quality index and serum prolactin and serum dehydroepiandrosterone sulphate levels in patients with chronic urticaria. Asian Pac J Allergy Immunol 2014; 32: 293-9.

28. Liu BJ, Yao ZJ, Si AL, et al. Life quality of Chinese patients with chronic urticaria as assessed by dermatology life quality index. J Eur Acad Dermatol Venereol 2012; 26: 1252-7.

29. Ucmak D, Akkurt M, Toprak G, et al. Determination of dermatology life quality index, and serum C-reactive protein and plasma interleukin-6 levels in patients with chronic urticaria. Adv Dermatol Allergol 2013; 30: 146-51.

30. Perkowska J, Kruszewski J, Gutkowski P, et al. Occurrence of sleep-related breathing disorders in patients with chronic urticaria at its asymptomatic or oligosymptomatic stages. Adv Dermatol Allergol 2016; 33: 63-7.

31. Evers AWM, Duller P, van de Kerkhof PCM, et al. The impact of chronic skin disease on daily life (ISDL): a generic and dermatology - specific health instrument. Br J Dermatol 2008; 158: 101-8.

32. Schmid-Ott G, Kuensebeck HW, Jaeger B, et al. Validity study for the stigmatization experience in atopic dermatitis and psoriatic patients. Acta Derm Venereol 1999; 79: 443-7.

33. Świnoga M. The assessment of selected psychological parameters in patients with contact dermatitis. PhD dissertation. UM, tódź 2014.

34. Alan S, Canan F, Karakas AA, et al. Temperament and character profiles of patients with chronic idiopathic urticaria. Postep Derm Alergol 2015; 32: 167-72.

35. Van Laarhoven AIM, Kraaimaat FW, Wilder-Smith OH, et al. Role of attentional focus on bodily sensations in sensitivity to itch and pain. Acta Derm Venereol 2010; 90: 46-50.

36. Evers AWM, Duller P, De Jong EMGJ, et al. Effectiveness of a multidisciplinary itch-coping training programme in adults with atopic dermatitis. Acta Derm Venereol 2009; 89: 57-63. 